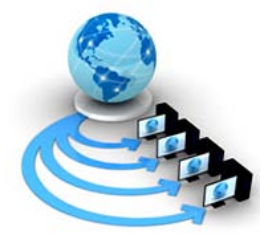

Volume 9, No. 2, March-April 2018

RESEARCH PAPER

Available Online at www.ijarcs.info

\title{
COCOWA MODEL: WATCHDOG MECHANISM USED TO PROPAGATE SELFISH NODES IN CELLULAR NETWORKS OF MOVING VEHICLES
}

\author{
R. Ragunathan \\ M.Sc Project Student \\ Department of Information Technology \\ Bharathiar University \\ Coimbatore-46, India
}

\author{
Dr. R. Vadivel \\ Assistant Professor \\ Department of Information Technology \\ Bharathiar University \\ Coimbatore- 46, India
}

\begin{abstract}
The cellular networks are used to move the rapid growth in the mobile data in the explosion on the cellular network. The offload cellular networks are dynamic in the swimming condition. The highly dynamic sector is improved by the WIFI networks the Access point is configured by the MAC and the IP address. The mobile Ad hoc network model is formatted on the selfish node behavior .The detecting selfish node is performed by the watch dog timers. The swimming performances are used to move the delay tolerant networks. The cocowa approach based on the diffusion on the selfish node propagated. The collaborative approach are reduces the time and increases the precision when detecting the selfish nodes.
\end{abstract}

Keywords: wireless construction, unicast, uplink and downlink, COCOWA.

\section{INTRODUCTION}

The pervasive computing systems, touching upon every aspect of life, will be partially supported by the sensor network infrastructure, which is involved in two processes: monitoring the surrounding environment, and providing information to analyze and respond. [1] On one hand, a sensor network may leak information about people to an unauthorized party, which leads to a privacy breaching. On the other hand, it may also lie about the collected data to a valid query making the network dysfunctional. [2] Unfortunately, little research work has targeted to solve the problem. The basic idea is that the network owner converts a top- $k$ query into another such that the query result for the converted query contains the true top- $k$ data items generated by legitimate sensor nodes with overwhelming probability.

The localization Requirement is fundamental in most sensor network applications and can be satisfied. There might be sensor nodes in the overlapping area of multiple cells, in which case they are affiliated with all those cells. Master and sensor nodes significantly differ in their resources. [3]. Since the operations in different cells are independent from each other, the adversary will not gain more from the collaboration of compromised master sensor nodes in different cells.[4] The adversary may launch different attacks through compromised $M$, sensor nodes, or both. From protocol execution, such that the network owner will falsely identify $M$ as malicious. , it do not intend to ensure data confidentiality against master nodes. [5]Many sensor network applications do not require data confidentiality but only query result Authenticity and soundness.

\section{RELATED WORK}

The wireless sensor nodes are monitored on the information traces. Nodes merged on the network management system on the organization.[6] The trace depend on the flow of frames. The higher intersections are metric in the similarity Method. The parameters are assigning on the incorporate similarity to the top ranked traces. [7]. The diverse the data to be the alternatives in the weight of the flow. The ranking methods are merged on the collected nodes. The fully connected graph is edged on the traces in the ranking similarity in the vertices. [8] The relevant rate is modified on the path of graph. The main impact on the ranking sector monitored on the bottom of the sensed badly deployed sensed nodes. [9]The nodes are monitored on the spontaneous wireless sensor nodes accessible.

The traces log is knowledge on the trace file. Every node is formatted on the logging sector. The iterative nodes are labelled in the structure of the memory management system. [10]The node deployed wireless sensor networks are depend on the performance for the routing protocol. The customize rate of data modified by the routed data. The sensor node randomized on the hoc manner. The energy efficient wireless sensor networks are the design the key factor to be challenged. The energy efficient model are minimize on the residual sensor nodes. [11]The energy balanced routing protocol are construct virtual potential field in the energy density. [12] The eliminate the loops in the simulation model. The fully balance routing protocol are used to eliminate the numerous driven deployment model. 


\section{METHODOLOGY}

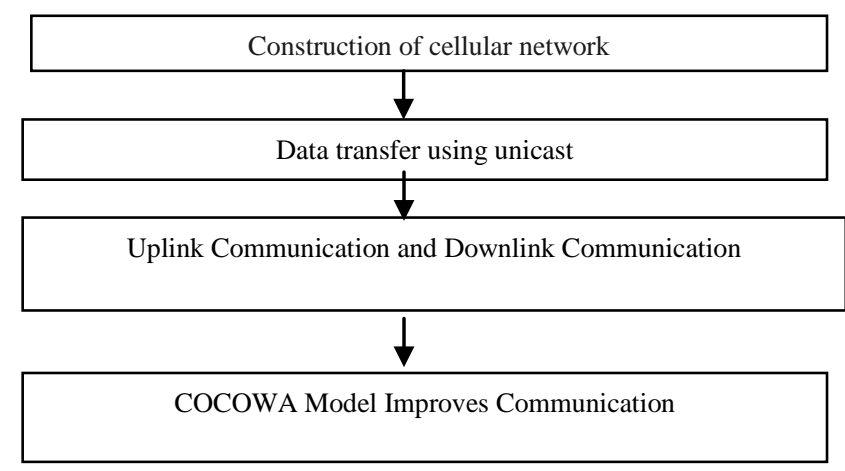

Fig. 1 Proposed Methodology

\section{A. Construction of cellular network}

A cellular network data transmission system including a digital communicator having a memory in which data is stored and an automatic dialer at a specific location for responding to an electrical signal indicating a specific condition exists, a cellular interface connected to the digital communicator and a cellular transceiver having an antenna, connected to the cellular interface, a cellular network including a central computer and an antenna for receiving signals from the transceiver antenna and for transmitting such signals from the central cellular computer site to a central monitoring station whereby the occurrence of a specific condition at the specific location initiates the dialing of an assigned number by the automatic dialer and the transmission of data corresponding to the specific location from the memory through the interface, transceiver and cellular network to the central monitoring station, the cellular network also adapted to receive signals initiated by the central monitoring station and to transmit such signals from the central cellular computer site to the transceiver antenna, whereby such signals are sent through the cellular network, transceiver and interface to the digital dialer and digital communicator.

\section{B. Data transfer using unicast}

Unicast is a one-to-one connection, meaning a connection from one node in a network to another node in a network. Unicast is the most used mechanism of sending data in Wireless networks as all normal UDP/TCP connections are one-to-one connections. Every packet has a certain identifier, so when the packet is received by the receiver, the receiver sends an acknowledgment back coming that this particular packet has been received. A packet is than 'lost', meaning that an acknowledgment is never send back. The sender thus never receives an acknowledgment and will try to send the packet again, until it receives an acknowledgment that the packet was received or until the Retry Count is met.

\section{Uplink Communication and Downlink} Communication

The transmission medium after this short interval is reserved for a period of ACK transmission. Because of this even if the simultaneously transmitted ACKs collide at the client, none of the other signals exist during that period when ACK is transmitted. Therefore, we can detect ACK even if collision happens.

\section{Downlink Communication}

Therefore for downlink communications a multicast group is established for each client. Downlink commutation can be executed in 2 stages.

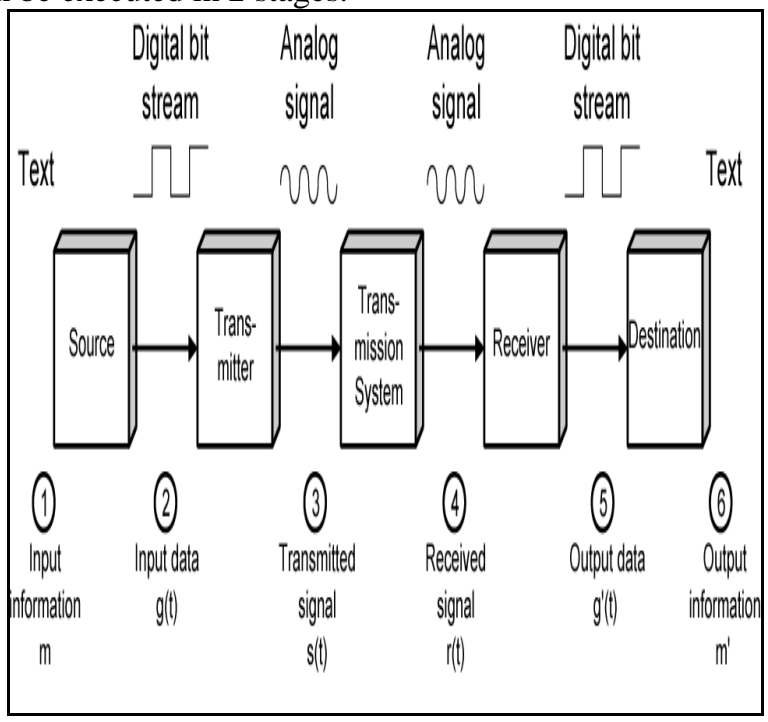

Fig. 2 Download Communication

\section{E. COCOWA Model Improves Communication}

The COCOWA model are used for the new method of the detecting the selfish node. The watchdog timer network is monitored on the detecting selfish node .This way, nodes have second hand information about the selfish nodes in the network.

\section{SIMULATION ENVIRONMENT}

This project is been developed using Java language and Java Netbeans. The performance evaluation is done in Java environment. NetBeans IDE is a free, open source, integrated development environment (IDE) that enables you to develop desktop, mobile and web applications. The IDE supports application development in various languages, including Java, HTML5, PHP and C++. The IDE provides integrated support for the complete development cycle, from project creation through debugging, profiling and deployment. The IDE runs on Windows, Linux, Mac OS X, and other UNIX-based systems.

\section{a. Initial configuration}

1. First run after installation will install the autoupdate plugin. Please do restart after installing the plugin.

2. Second run will find a number of updates which you are advised to install.

3. Go to Tools $>$ Plugins menu

4. Switch to the "Available plugins..." tab

5. Look for a plugin with the name Subversion in the Base IDE category

6. Select it by checking the checkbox to the left and click on the Install button at the bottom of the Plugins dialogue

7. Restart after the installation is done and you now have a subversion client within your IDE.

8. The start page of NetBeans consisting of related news can be turned off by unchecking the "Show on Startup" checkbox at the bottom of the screen. 
Table 1: Simulation Parameters

\begin{tabular}{|c|c|c|c|}
\hline \multicolumn{2}{|c|}{ Software requirements } & \multicolumn{2}{|c|}{ Hardware requirements } \\
\hline IDE & Net beans & Processor & $\begin{array}{l}800 \mathrm{MHz} \\
\text { Intel } \\
\text { Pentium III } \\
\text { or } \\
\text { equivalent }\end{array}$ \\
\hline $\begin{array}{l}\text { Developer } \\
\text { tool }\end{array}$ & $\begin{array}{l}\text { Java SE } \\
\text { Development } \\
\text { Kit (JDK) }\end{array}$ & Memory & $500 \mathrm{~GB}$ \\
\hline $\begin{array}{l}\text { Operating } \\
\text { system }\end{array}$ & Windows & RAM & $1 \mathrm{~GB}$ \\
\hline $\begin{array}{l}\text { Java } \\
\text { Library }\end{array}$ & $\begin{array}{l}\text { JavaFX } \\
\text { Support }\end{array}$ & $\begin{array}{l}\text { Monitor } \\
\text { Keyboard }\end{array}$ & $\begin{array}{l}\text { LED 16” } \\
\text { Multimedia }\end{array}$ \\
\hline Database & JavaDB & & $\begin{array}{l}\text { Keyboard } \\
\text { with }\end{array}$ \\
\hline Language & Java EE & & $\begin{array}{l}\text { wireless } \\
\text { mouse }\end{array}$ \\
\hline
\end{tabular}

\section{PERFORMANCE EVALUATION}

In this result we use to compare Watchdog, and Swimming experiments like Execution time, throughput. The watchdog execution strategy was less time consumed. The watchdog throughput is finally improved.

Table 2. Execution time

\begin{tabular}{|l|l|}
\hline Algorithm Name & Metrics \\
\hline Watchdog & 3 \\
\hline Swimming & 7 \\
\hline
\end{tabular}

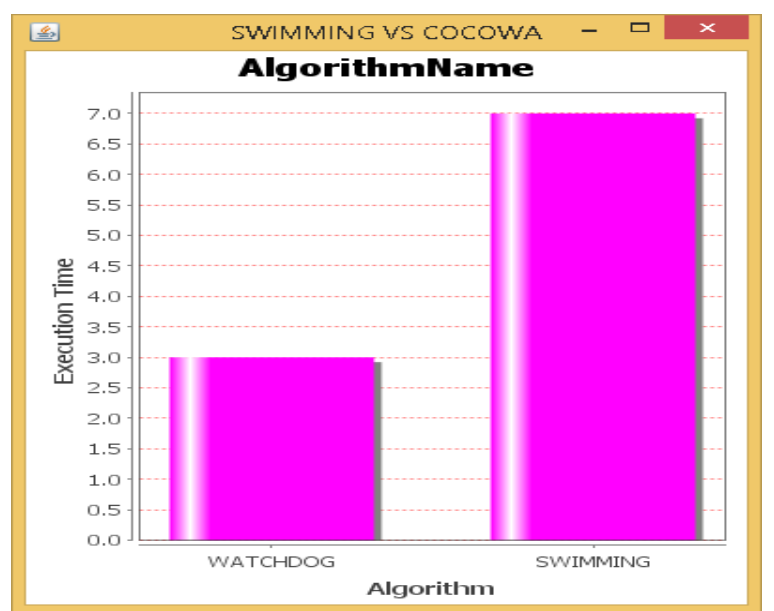

Fig 3: The Watchdog has been compared to Swimming

Table 3. Throughput

\begin{tabular}{|l|l|}
\hline Algorithm name & Throughput \\
\hline Watchdog & 0.012 \\
\hline Swimming & 0.003 \\
\hline
\end{tabular}

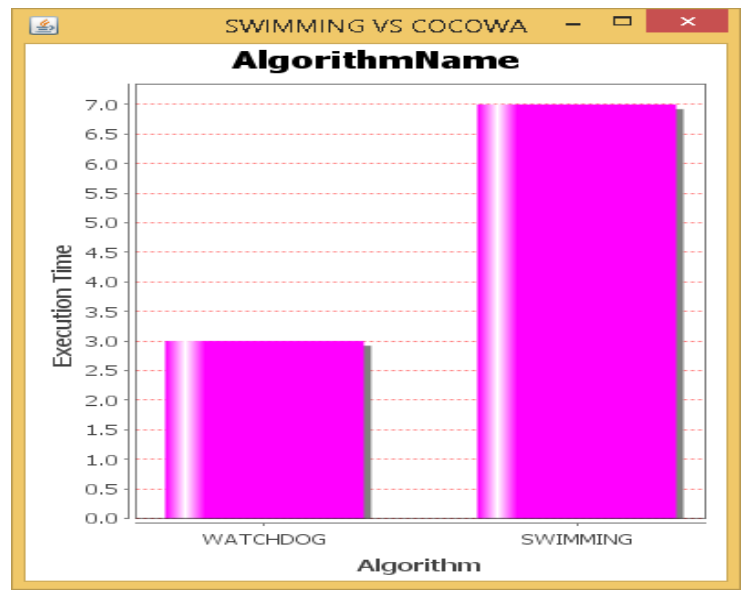

Fig. 4 Swimming has been compared to Watchdog

\section{CONCLUSION}

The proposed approach prolongs the unicast data using download and uploads links of the WSN system. The watchdog mechanism performs well in wireless network. It is used to improve the throughput and reduce execution time.

\section{REFERENCES}

[1] P. A. K. Acharya, A. Sharma, E. M. Belding, K. C. Almeroth, S. Member, and D. Papagiannaki, "Rate adaptation in congested wireless networks through real-time measurements," IEEE Transactions on Mobile Computing, vol. 9, no. 11, pp. 1535-1550, Nov. 2010.

[2] Y. Sheng, G. Chen, H. Yin, K. Tan, U. Deshpande, B. Vance, D. Kotz, A. Campbell, C. McDonald, T. Henderson, and J. Wright, "MAP: a scalable monitoring system for dependable 802.11 wireless networks,” Wireless Communications, IEEE, vol. 15, no. 5, pp.10-18, October.

[3]. K. Tan, C. McDonald, B. Vance, C. Arackaparambil, S. Bratus, and D. Kotz, "From MAP to DIST: the evolution of a largescale WLAN monitoring system,” IEEE Transactions on Mobile Computing, vol. 99, no. PrePrints, p. 1, 2012.

[4] Matteo Sammarco, Miguel Elias M. Campista, and Marcelo Dias de Amorim "Scalable Wireless Traffic Capture Through Community Detection and Trace Similarity” in IEEE TRANSACTIONS ON MOBILE COMPUTING vol.15, issue July 2016

[5] U. N. Raghavan, R. Albert, and S. Kumara, "Near linear time algorithm to detect community structures in large-scale networks," Physical Review E, vol. 76, no. 3, pp. 036 106+, Sep. 2007. [Online].

Available: http://dx.doi.org/10.1103/physreve. 76.036106

[6] A. Argyriou and V. Madisetti, "Using a New Protocol to Enhance Path Reliability and Realize Load Balancing in Mobile Ad Hoc Networks,” Ad Hoc Networks, vol. 4, pp. 60-74, 2006.

[7] A. Bletsas, A. Khisti, D.P. Reed, and A. Lippman, "A Simple Cooperative Diversity Method Based on Network Path Selection,” IEEE J. Selected Areas in Comm., vol. 24, no. 3, pp. 659-672, Mar. 2006.

V. Venkataramanan, X. Lin, L. Ying, and S. Shakkottai, "On Scheduling for Minimizing End- 
to-End Buffer Usage over Multi- Hop Wireless Networks," Proc. IEEE INFOCOM, 2010.

[9] B. B. Romdhanne, D. Dujovne, and T. Turletti, "Efficient and scalable merging algorithms for wireless traces," in Workshop on Real Overlays and Distributed Systems (ROADS), Oct. 2009, pp. $1-7$.

[10] A. Balachandran, G. M. Voelker, P. Bahl, and P. V. Rangan, "Characterizing user behavior and network performance in a public wireless LAN," in ACM SIGMETRICS, Jun.15-19 2002, pp. 195-205.
[11] M. Sammarco, M. E. M. Campista, and M. D. de Amorim, "Trace selection for improved WLAN monitoring," in ACM HotPlanet Workshop, Aug.16 2013, pp. 1-6.

[12] M. E. J. Newman and M. Girvan, "Finding and evaluating community structure in networks," Physical Review E, vol. 69, no. 2, pp. 026 113+, Feb. 2004. [Online]. Available: http: //dx.doi.org/10.1103/physreve.69.02611. 